\title{
The measuring technique developed to evaluate the thermal diffusivity of the multi-layered thin film specimens
}

\author{
Tse-Chang $\mathrm{Li}^{1, *}$, Yang-Ru $\mathrm{Li}^{1}$, and Jen-Fin $\mathrm{Lin}^{1}$ \\ ${ }^{1}$ Department of Mechanical Engineering, National Cheng Kung University, Tainan 701, Taiwan
}

\begin{abstract}
In the present study, the thermal diffusivities of the Al, Si and ITO films deposited on the SUS304 steel substrate are evaluated via the present technique. Before applying this technique, the temperature for the thin film of the multi-layered specimen is developed theoretically for the onedimensional steady heat conduction in response to amplitude and frequency of the periodically oscillating temperature imposed by a peltier placed beneath the specimen's substrate. By the thermal-electrical data processing system excluding the lock-in amplifier, the temperature frequency $a_{3}$ has been proved first to be independent of the electrical voltage applied to the peltier and the contact position of the thermocouples. The experimental data of phase difference for three kinds of specimen are regressed well by a straight line with a slope. Then, the thermal diffusivity of the thin film is thus determined if the slope value and the filmthickness are available. In the present arrangements for the thermocouples, two thermal diffusivity values are quite close each other and valid for every kind of specimen. This technique can provide an efficient, low-cost method for the thermal diffusivity measurements of thin films.
\end{abstract}

\section{Introduction}

Measurement of thermal diffusivity in diversity materials had been studied for various sizes of samples. Recently, much attention had been paid to the technique for the samples with multi-layer thin films. Apart from the intrinsic importance of thermal diffusivity, its determination gives the value of the thermal conductivity $(\mathrm{k})$, if the material density $\rho$ and the thermal capacity at constant pressure $\left(\mathrm{C}_{\mathrm{p}}\right)$ are available.

A simple apparatus was described in the studies of Asams and Kirkbright $[1,2]$ that permitted heat-flow characteristics of a sample to be determined, and its application of the thermal diffusivity or thickness of polymer films on a copper substrate was reported. The amplitude and phase of observed optoacoustic signal depend on the thermal diffusivity and thickness of the material irradiated. The use of time-resolved temperature measurement techniques for the evaluation of the thermal diffusivity of material had been documented, and most methods were based on the original work undertaken by Ångström [3] and later modification by King [4] and Abeles et al. [5]. Development of the phase-lag methods for use with thin slabs or discs of material had been reviewed by Touloukian et al. [6].

There had been a few experiments using an ac calorimetric method on thermal diffusivity so far $[7,8]$. A method to measure thermal diffusivity of thin sample no matter how thin a sample may be. It was useful in the measurement for thin materials with a variety of thermal diffusivities. A variation of the thermal conductance measurements was described using the dynamic $3 \omega$ method [9]. By using the $3 \omega$ method, the measurements of temperature oscillation needed to be obtained and calculated. Thus, the experimental data would taken into account the thermal resistance between the sample and the thermocouples and the heat capacity of the contact point of the thermocouples. In order to use this method, a fixed sample geometry should be provided. Simple temperature oscillation techniques were adopted in the study of Czarnetzki and Roetzel [10] to describe for the fast measurement of thermal diffusivity and thermal conductivity of liquids. The thermal diffusivity and conductivity of the specimen were deduced by measuring and evaluating the amplitude attenuation and/or the phase shift between the fundamental temperature oscillations at the surface of the liquid specimen and a well-defined position inside the specimen. The results agreed well with the data obtained from the literature.

An experimental technique, namely the amplitude method, was proposed in the study of Zhang and Grigoropoulos [11], and applied to measurement of the thin film thermal diffusivity. The thermal diffusivity was determined by three independent experimental methods. Good agreement among the measured thermal diffusivities obtained by the three methods confirmed the validity of the amplitude method. The work of $\mathrm{Yu}$ et al. [12] investigated and compared three laser-based ac heating methods for the thermal diffusivity measurement of free-standing thin film structures. Both the phase and the amplitude signals of the ac temperature rise of the sample were ultilized to deduce the thermal diffusivity of the thin film structures. The results showed that the

Corresponding author: TCL92@mail.ncku.edu.tw 
uniform illumination method was more susceptible to the error than the other two configurations due to twodimensional effects. In the study of Jansen and Obermeier [13], an accurate technique to measure the thermal conductivity of the thin films in a wide temperature range was presented. Measurements on polycrystalline diamond films due to the variations of thermal conductivity with temperature had been explained by the effect of the small grain size of the diamond film. Experimental and theoretical studies of heat transport by lattice vibrations in thin film materials were reviewed [14]. Selected examples of experimental methods are discussed to illustrate a variety of methods that have been developed for thin film measurements. Dielectric thin films have significant atomic-scale disorder, and the heat transport is dependent upon this disordered material. The study of Bhusari et al. [15] presented the design of a simple setup for measuring the thermal conductivity of thin films. This method was based on the principle of phase lag of a traveling thermal wave. The phase lag $(\Delta \theta)$ between the excitation wave and resulting thermal wave, at a variable distance $\mathrm{d}$ from the edge of the sample, was determined by measuring the deflection of another "probe-laser". This method offered an accuracy of better than $\pm 0.5 \%$. The thermal diffusivities of thin films of evaporated metal less than $1 \times 10^{-6} \mathrm{~m}$ thick had been measured in the study of Yamane et al. [16] using the light-irradiation ac calorimetric method. The thermal diffusivities of metallic thin films become smaller than those of the bulk material as the thickness of the metallic thin films decreased. In the study the Chung et al. [17], the photothermal deflection technique is used to measure the thermal diffusivity of a metal-ceramic decomposite. The Kapitza radius is used as the effective value of the thermal boundary resistance of heterointerfaces. Possible reasons for the data from metal-ceramic composite materials were discussed in terms of the acoustic mismatch model for thermal boundary resistance. A picosecond thermoreflectance measurement system had been developed in the study of Taketoshi et al. [18] in order to measure thermal diffusivities of metal thin films. In-plane thermal diffusivities of the thin films are close to those of the bulk materials although out-of-plane electrical resistivities measured by the four-probe method were larger than that of the bulk materials. The development of an approach to the Angstrom's method for the measurement of the thermal diffusivity was reported in the study of Muscio et al. [19]. In this method, the diffusivity is determined from the damping and the phase shift of a periodic thermal signal during its propagation along the specimen. A direct-contact source was employed, with a temperature-oscillation signal supplied on a portion of one of the two main surfaces of the specimen. The main difficulty about its use is to obtain a perfectly harmonic and well-balanced thermal signal. Photothermal beam deflection technique had been employed for the measurement of thermal diffusivity of different compositions of amorphous $\mathrm{Ge}_{\mathrm{x}} \mathrm{Se}_{100-\mathrm{x}}$ thin films [20]. Slope from the tangetial component of the deflection signal with the pump-probe offset was used to evaluate the numerical value of thermal diffusivity. The thermal diffusivity and conductivity of the EB-PVD $7 Y S Z$ thermal barrier coating were evaluated on the basis of its photothermal infrared emission [22, 23]. Photopyroelectric spectroscopy (PPE) was used to study the thermal and optical properties of melanins. The photopyroelectric intensity signal and its phase were independently measured as a function of wavelength and chopping frequency for a given wavelength in the saturation part of the PPE spectrum. The thermal diffusivity coefficient, thermal conductivity, the specific heat of the samples, and a value for the condensed phase optical gap could be obtained [24]. Thermal properties, such as thermal conductivity, thermal diffusivity, thermal effusivity and specific heat capacity, of geometrically frustrated multiferroic $\mathrm{ErMnO}_{3}$, having range of grain sizes from 32 to $220 \mathrm{~nm}$ has been measured by the photopyroelectric technique [25]. The coatings at high-temperature exposure with thermal cycling brought in substantial increases in thermal diffusivity and conductivity. The thermal conductivity of the films was measured using the dynamic $3 \omega$ method [9]. This method was employed to measure thermal conductivity of $\mathrm{Ni}, \mathrm{Co}$, and Fe-doped $\mathrm{La}_{5} \mathrm{Ca}_{9} \mathrm{Cu}_{24} \mathrm{O}_{41}$ thin films.

The thermal conductivity of multi-layer composites can differ markedly from that of the corresponding bulk materials. Many methods for the measurement of the thermal conductivity of multi-layer composites have been reported [26-28]. A simple arrangement of doublelayer composites has the component materials arranged as parallel slabs. In such composites, the thermal conductivities have been reported for the two extreme cases of heat flow parallel and perpendicular to the plane of the slabs. Multilayers consisting of transition-metal silicides have been achieved by the size of growing silicides strongly depends on the annealing conditions [29].

The advantages of the present measurements are: (1) the thermal diffusivity $(\alpha)$ is determined dependent upon the film thickness and the oscillating temperature frequency $(\omega)$ only, stable and accurate measures can be obtained from the measurements so long as the $\omega$ parameter is proved to be independent of the thermocouples contact position and the electrical voltage applied to the peltier; (2) the specimens can be prepared in a simple geometry with multilayer films; (3) the components in the thermal-electrical detecting system are low cost and thermal diffusivities of all layers can be achieved in just one specimen and simple way compared to the other techniques. In the present study, the phase lag method is adopted to evaluate the thermal diffusivity of each layer of two multi-layered specimens. A container of multi-layered specimen with a property of good heat insulation at the specimen's lateral surfaces is appropriately designed with a ventilation system placed beneath the peltier such that the oscillating temperature at everywhere of the specimen is presented to be uniform and operating at the steady condition. The data processing system applied to the temperature signals of the three thermocouples is composed of a data acquisition system and a lock-in amplifier. The theoretical temperature solution in the multi-layered 
specimens is developed for one-dimensional, oscillating heat conduction in terms of the amplitude and frequency of the oscillating temperature imposed by a peltier placed beneath the specimen's substrate. The effects of electrical voltage applied to the peltier and different thermocouple contact position on the frequency of the periodically oscillating temperature at various layers are first evaluated using the thermal-electrical data processing system excluding the lock-in amplifier. Theoretically, different phase lag in temperature exists at every layer of the specimen w.r.t the oscillating temperature of the peltier. The difference in the phase lag between the top and bottom surfaces of every layer can be expressed as a function of layer thickness (d), oscillating temperature frequency $(\omega)$, and thermal diffusivity $(\alpha)$ of this layer. Two of three thermocouples are placed to have their tip in contact with the top surface of the thin film at arbitrary positions in order to achieve the phase lags occurring at two different positions of this layer. The third thermocouple is placed on the top surface of the lower layer adjacent to this measured film in order to evaluate the temperature phase difference across this thin film with a thickness of $d$. The thermal-electrical data processing system with a lock-in amplifier was then used to obtain the temperature solution as a function of time. The phase difference between any two of these three thermocouples can be measured as a function of wave frequency $(\omega)$ in a form of $a+b \sqrt{\omega}$. The differentiation of this phase difference w.r.t. $\sqrt{\omega}$ is obtained equal to $\mathrm{d} / \sqrt{2 \mathrm{a}}(=\mathrm{b})$. The thermal diffusivity $(\alpha)$ of the film layer is thus determined if the $\mathrm{b}$ value (slope of the straight line) and the measured film thickness $d$ are available. The thermal diffusivities of three thin film materials determined by the present technique are then compared with those reported in the literatures. The effect of the surface roughness on the measures of thermal diffusivity is investigated.

\section{Theoretical models for thermal diffusivity}

In order to measure the thermal diffusivity of each layer in the multi-layered specimen, the container of the specimen should be properly designed to have good thermal insulation such that the heat transfer in the specimen can be to the utmost the one-dimensional heat conduction. The temperature at every layer of specimen is regarded to be uniformly distributing but oscillating with time in a thermally stable form if one-dimensional heat transfer is assumed. If the one-dimensional heat transfer is satisfied, it can be obtained as

$$
\frac{\partial^{2} \mathrm{~T}}{\partial \mathrm{x}^{2}}=\frac{1}{\alpha} \frac{\partial \mathrm{T}}{\partial \mathrm{t}}
$$

The $\mathrm{x}$-axis is defined to be normal to the specimen and in the upward direction, with its origin point set at the interface of the measured film and its adjacent lower layer. In the present study, a peltier is placed beneath the substrate of the multi-layered specimen. The heat produced by the peltier is released in an oscillating form with a frequency $\omega\left(=2 \pi / t_{\mathrm{p}} ; \mathrm{t}_{\mathrm{p}}\right.$ : period). Fins and fan are placed in sequence beneath the peltier. The composition of the specimen + peltier + heat ventilation system is then held firm by the container made of Acrylic material. It should be mentioned that this material is a good insulator with a thermal conductivity of 0.2 $\mathrm{W} \cdot \mathrm{m}^{-1} \cdot \mathrm{K}^{-1}$ only. Air was sucked into the chamber (see Fig. 1) by the fan to cool the fins protruding out of the bottom. Appropriate design for the air flow rate provides the temperature variations in the peltier to be oscillating but in a stable condition; that is, the temperature oscillations are operating in a stable band almost invariant with time. If so, the heat transfer in the multilayered specimen can be regarded to be one-dimensional at every layer. Define $\xi \equiv \mathrm{x}\left(\frac{\omega}{2 \alpha}\right)^{1 / 2}$ and $\tau \equiv \omega \mathrm{t} . \xi$ is then specified as the dimensionless phase lag of the oscillating temperature arising at specimen's layer. Define $\mathrm{d}$ as the thickness of thin film (or substrate). $\xi \mathrm{d} \leq 0.1$ is the additional prerequisite of onedimensional heat transfer in the multi-layered specimens. The dimensionless form of Eq. (1) is then rewritten as:

$$
\frac{\partial^{2} \mathrm{~T}}{\partial \xi^{2}}=2 \frac{\partial \mathrm{T}}{\partial \tau}
$$

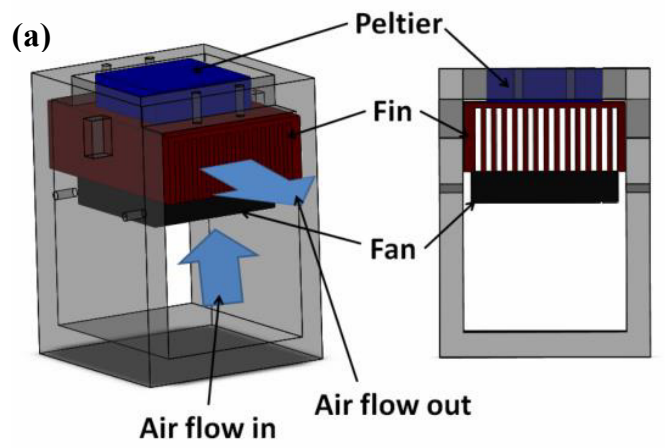

(b)

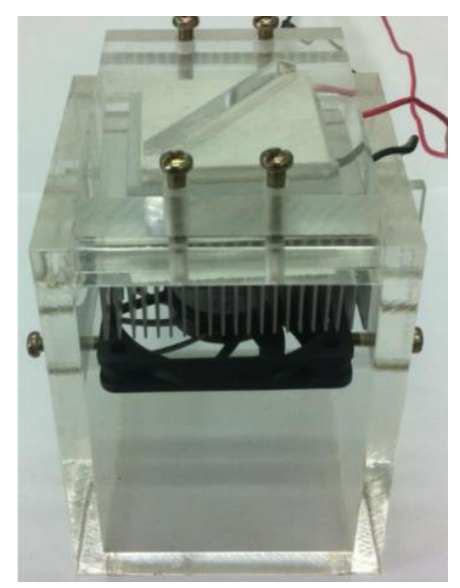

Fig. 1. (a) Ventilation and cooling system for peltier; (b) the photograph of the specimen heating system. 
The temperature solution of Eq. (2) is obviously obtained as a function of time. Define the $\mathrm{x}$-axis in the direction normal to the thin film top surface, and $x=0$ is positioned at the bottom surface of the measured film. If the heat transfer is controlled to be one-dimensional in the $\mathrm{x}$-direction, the temperature solution in an oscillating form with a fixed frequency $\omega$ is almost uniformly distributing over every cross section of the multi-layered specimen. With this condition, Eq. (2) is conveniently considered as the quasi-steady state, and the solution of the film layer with the factor of time is obtained simply from the boundary condition imposed on the bottom surface of the layer in an oscillating form. Due to the complicate geometries of the multi-layered specimen, it is indeed difficult to attain the temperature solution in an analytical form for the entire specimen. Instead of this, the analytical solution of temperature is now established for every individual layer by imposing appropriate boundary conditions for the top and bottom surfaces of this layer. For example, Figure 2 shows film A deposited on the substrate with $d$ as the thickness. The coordinate set for film A is shown in Fig. 2. The boundary conditions given for the bottom and top surfaces of film A are [10]:

$$
\begin{aligned}
& \xi=0, \quad T(x=0, \tau)=T_{M}+u_{0} \cos \tau \\
& \xi=\infty, \quad \frac{\partial T}{\partial \xi}=0
\end{aligned}
$$

\section{Heat transfer direction}

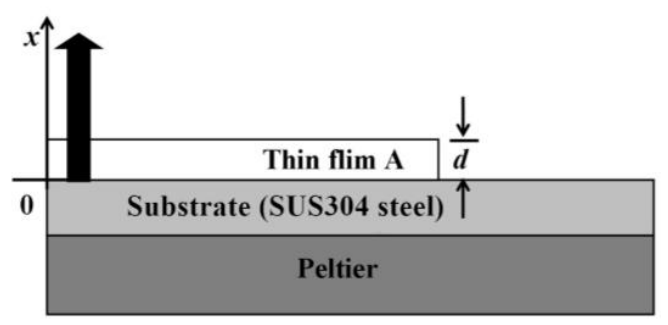

Fig. 2. The schematic diagram of the multilayered specimen with its origin point located at the interface of film A and substrate.

where $T_{M}$ denotes the mean value of the oscillating temperature occurring at the interface of the substrate and film $\mathrm{A} ; \mathrm{u}_{0}$ denotes the amplitude of the oscillating temperature. $\mathrm{T}_{\mathrm{M}}$ and $\mathrm{u}_{0}$ are actually two unknowns in the current situation. Solution of Eq. (2) satisfying these two boundary conditions is thus expressed as [10]

$$
\mathrm{T}(\xi, \tau)=\mathrm{T}_{\mathrm{M}}+\mathrm{u}_{0} \mathrm{e}^{-\xi} \cos (\tau-\xi)
$$

$\xi$ denotes the phase difference in temperature w.r.t. the oscillating heat transfer at $\xi=0$. It should be mentioned that this heat transfer is actually unknown. In the present study, a reference signal corresponding to a fixed electrical voltage (10 $\mathrm{V}$ in this study) but a variable frequency was given. Then, the phase difference w.r.t. the reference signal can be obtained for all three thermocouples. Then, the phase difference $\phi$ of temperature wave across the film with a thickness of $d$, according to the $\xi$ definition, is written as

$$
\phi=d \sqrt{\frac{\omega}{2 \alpha}}
$$

It should be mentioned that Eq. (5) is valid for the case that the top surfaces of all layers are prepared to be perfectly smooth such that the temperature measurement is irrelevant to the measuring position of the same surface.

\section{Experimental details}

The system for thermal diffusivity $(\alpha)$ measurement is composed of one-dimensional steady heat transfer design for multi-layered specimen and peltier's fan cooling system, the circuit control system for oscillating temperature amplitude and phase, thermocouples, and the lock-in amplifier for the grasps of the temperature signals with specified frequency and phase via reference signal. The schematic diagram of the system for the measurement of thermal diffusivity $(\alpha)$ is shown in Fig. 3. A lock-in amplifier (NI-4431, National Instruments, USA) is used to detect and measure very small AC signals all the way down to a few nanovolts. Accurate measurements may be made even when the small signal is obscured by noise sources many thousands of times larger. The lock-in amplifier uses a technique known as phase-sensitive detection to single out the component of the signal at a specific reference frequency and phase. Noise signals, at frequencies other than the reference frequency, are rejected and do not affect the measurement. A NE555 chip collaborating with the peltier was used for the controls of the oscillating temperature amplitude and frequency of peltier in an approximately sinusoidal form. Its circuits are adjustable for electrical capacitance and resistance to create various oscillating signals in a square form. An approximately sinusoidal temperature oscillation is thus created in the peltier. Beside the NE555 chip, a capacitor and a variable resistor are provided such that the product value of the electrical resistance (R) and capacitance (C) in a wide range is ensured to be linearly proportional to the oscillation period/frequency.

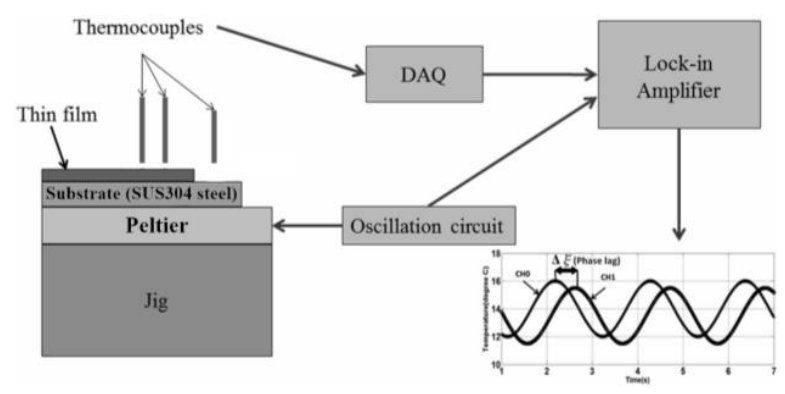

Fig. 3. Schematic diagram of the entire thermal-electrical system for the measurements of thin film thermal diffusivity.

As Fig. 3 shows, the multi-layered specimen is placed on the top surface of peltier in order to accept the 
oscillating heat waves uniformly distributing over the substrate-peltier interface. Uniform distribution of heat waves in the substrate is important in order to sustain the heat transfer in the multi-layered specimen to be considerably one dimensional. In order to achieve this goal, the jig for the peltier placement, as Fig. 1 shows, is designed to have a fence surrounding the lateral surfaces of the square peltier. The jig is made of the Acrylic material such that the temperature distribution on the peltier top surface is nearly uniform after heating for a while. On another hot surface of the peltier, the heat was dissipated by a row of fins and a fan installed for air ventilations. The peltier was provided as a cooling heat source to create an oscillating temperature wave. The choice of this kind of peltier is made for the purpose of achieving the specimen temperature a rapid thermal equilibrium with the environment. Fig. 1(b) shows the photograph of the peltier heating equipment, and a specimen has been placed on the top surface of the peltier. In the present study, each of all specimens is prepared to be a ladder form in a small area near the specimen edge. That is, there is a small area of the substrate surface without coating thin film. Three K-type thermocouples (AWG 40 ANSI, USA) with a temperature resolution of $\pm 1.1{ }^{\circ} \mathrm{C}$ were used as the temperature measurements at the top surfaces of the measured film and the substrate. Two thermocouples were placed to have contacts with the top surface of the measured film at arbitrary positions in order to examine the surface roughness effect on the phase of temperature waves. The third thermocouple was in contact with the blank area of the lower layer adjacent to the measured film. The temperature wave signals from these three thermocouples were acquired by a NI-4431 data acquisition system with a high-speed USB carrier (NI, USA); the synchronous sampling rate is up to $102 \mathrm{kS} / \mathrm{s}$. A "LabView" software was used to have a collocation with the data acquisition system to form the lock-in amplifier for the original temperature signals in a random form from these thermocouples.

\section{Results and discussions}

The frequency of oscillating temperatures is controlled by the peltier. Therefore, the temperature period $\bar{T}$ is governed by the product value of electrical resistance $(\mathrm{R})$ and electrical capicity (C) applied in the peltier controller. The relationship between the $\bar{T}$ and $\mathrm{R} \cdot \mathrm{C}$ parameters is provided in Fig. 4(a). A linear behavior is demonstrated in the period range used in this study. It implies that the temperature period, ant thus the period can be determined from $\bar{T}=1.635 \mathrm{R} \cdot \mathrm{C}$ if the $\mathrm{R} \cdot \mathrm{C}$ value applied to the peltier is available. Then, the frequency of oscillating $\omega$ is defined as $1 / \bar{T}$ and the period can be determined from $\omega=0.6581 / \mathrm{R} \cdot \mathrm{C}$ in Fig. 4(b).

Due to the steady oscillating temperature output from the peltier, the temperature (T) data measured by the thermocouples with their tip placed on the coating film and its substrate surface are able to express in a general form of $T(t)=a_{0}+a_{2} \cos \left(a_{3} t+a_{4}\right)$, where $t$ represents the time of heat conduction. The general $\mathrm{T}(\mathrm{t})$ solution is obtained as the sum of the constant and Cosine terms. The linear term $\left(a_{1} t\right)$ is neglected here if the peltier is controlled without the linear temperature rise arising at the thermocouples shown in Fig. 5(a). Figure 5(b) shows the temperature data acquired from these two thermocouples respectively. Since these data are presented without a linear increase with time, $\mathrm{T}(\mathrm{t})$ solution is simplified as $\mathrm{T}=\mathrm{a}_{0}+\mathrm{a}_{2} \cos \left(\mathrm{a}_{3} \mathrm{t}+\mathrm{a}_{4}\right)$. The data obtained from the $\mathrm{CH} 0$ for the substrate can be regressed well as $\mathrm{T}=15.29-1.773 \cos (0.7328 \mathrm{t}+0.0424)$; whereas the data form the $\mathrm{CH} 1$ for the Si film can be regressed as $\mathrm{T}=15.97-1.651 \cos (0.7328 \mathrm{t}+0.0572)$.
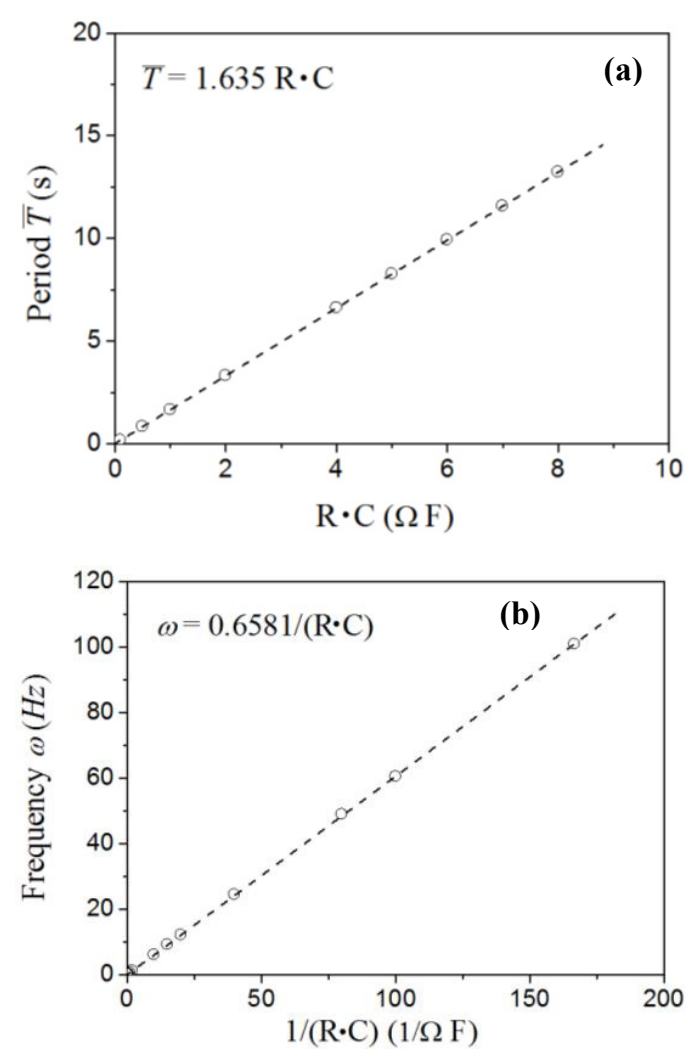

Fig. 4. Results of the time period (a) and frequency (b) of oscillating temperature expressed as a function of the product value of electrical resistance and capacity.

The $\mathrm{a}_{3}$ parameter in the $\mathrm{T}(\mathrm{t})$ expression represents the frequency of oscillating temperature, and $\mathrm{a}_{4}$ denotes the phase lag arising at the measured layer with a distance from the peltier. In order to investigate the variations of the $a_{3}$ parameter due to the changes in the electrical voltage and the measured point at the same surface, the thermal-electrical system was applied intently without the lock-in amplifier. The SUS304 steel and Si (200 $\mathrm{nm}$ )/SUS304 steel specimens are prepared for the temperature measurements. Two thermocouples are arranged to contact with either the SUS304 steel substrate or the peltier simultaneously at different contact positions. The $\mathrm{T}(\mathrm{t})$ expression is thus applicable to evaluate the temperature arising at different position. Then, the $a_{3}$ values associated with the above two 
thermocouple arrangements can be thus obtained from these temperature solutions.

(a)

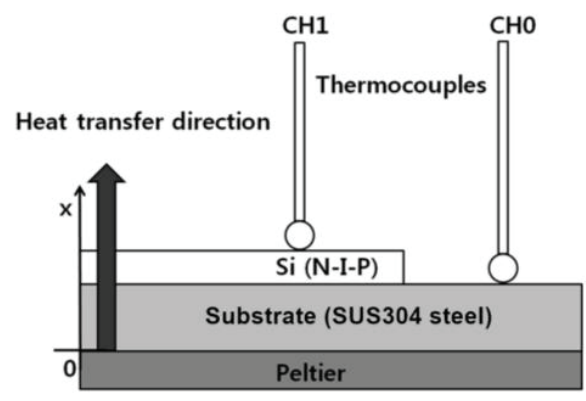

(b)

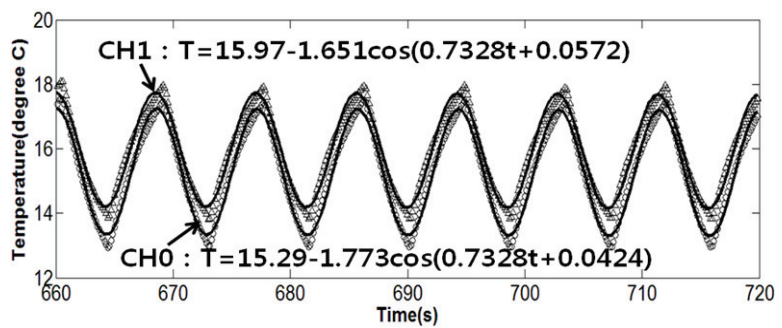

Fig. 5. (a) Schematic diagram of the thin film specimen heated by the peltier and the temperature at different layer detected by the $\mathrm{CH} 1$ and $\mathrm{CH} 0$ thermocouples; (b) the experimental data of the temperatures obtained from these two thermocouples and their regression.

Figure 6(a) shows the $a_{3}$ values obtained from the arrangements of these two thermocouples noted by $\mathrm{CH} 0$ and $\mathrm{CH} 1$ at two peltier oscillating frequencies and measuring at two arbitrary positions of the SUS 304 steel substrate. $a_{3} \cong 0.586 \mathrm{~Hz}$ is obtained from the measurements with peltier oscillating frequency of 0.586 $\mathrm{Hz}$; whereas $\mathrm{a}_{3} \cong 100.552 \mathrm{~Hz}$ is obtained from the measurements of the two thermocouples by applying peltier's oscillating frequency at $100.552 \mathrm{~Hz}$. It is interestingly found that the $a_{3}$ data obtained from the $\mathrm{CHO}$ and $\mathrm{CH} 1$ are quite close each other and nearly a value invariant with the measuring position and the peltier's oscillating frequency. The $\mathrm{Si}$ film was then deposited on the SUS304 steel substrate as the coating layer. The thermocouple labelled as $\mathrm{CHO}$ was placed in contact with the top surface of the SUS304 steel substrate; whereas the thermocouple noted by $\mathrm{CH} 1$ was placed on the top surface of the Si film. The $\mathrm{a}_{3}$ values obtained from these two thermocouples at different positions are shown in Fig. 6(b). Theses data can be regressed well with $a_{3} \cong 0.586 \mathrm{~Hz}$ in response to the peltier's oscillating frequency of $0.586 \mathrm{~Hz}$, and $\mathrm{a}_{3}$ $\cong 100.552 \mathrm{~Hz}$ is obtained as the applied oscillating frequency is $100.552 \mathrm{~Hz}$. The provisions of Fig. 6(a) and Fig. 6(b) for the $a_{3}$ results can prove that: (1) $a_{3}$ is a constant value nearly invariant with the contact positions of two thermocouples placed at the same surface; (2) $a_{3}$ is a constant value invariant with the contact positions at two different contact surfaces; (3) $a_{3}$ is obtained very close to the peltier's oscillating frequency. It is thus concluded that the frequency of oscillating temperature in the peltier is sustained to be almost unchanged in the heat conduction for any layer of the specimen. Therefore, the $a_{3}$ parameter in the $T(t)$ expression is regarded as a constant in the solution of thermal diffusivity for any coating film, irrespective of the contact position of thermocouples.

(a)

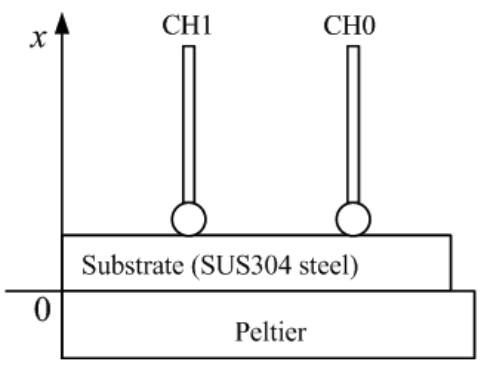

\begin{tabular}{|c|c|c|c|c|c|c|}
\hline \multicolumn{2}{|c|}{$\begin{array}{l}\text { Different } \\
\text { positions } \\
\text { in peltier }(\mathrm{Hz})\end{array}$} & 1 & 2 & 3 & 4 & 5 \\
\hline \multirow{2}{*}{0.586} & $\mathrm{CH} 0$ & 0.58625 & 0.58528 & 0.58675 & 0.58588 & 0.58674 \\
\hline & $\mathrm{CH} 1$ & 0.58703 & 0.58635 & 0.58612 & 0.58647 & 0.58549 \\
\hline \multirow{2}{*}{100.552} & $\mathrm{CH} 0$ & 100.552 & 100.552 & 100.554 & 100.555 & 100.556 \\
\hline & $\mathrm{CH} 1$ & 100.553 & 100.555 & 100.553 & 100.555 & 100.554 \\
\hline \multicolumn{2}{|c|}{ Aplied $\omega$} & 6 & 7 & 8 & 9 & 10 \\
\hline \multirow{2}{*}{0.586} & $\mathrm{CH} 0$ & 0.58519 & 0.58688 & 0.58703 & 0.58635 & 0.58612 \\
\hline & $\mathrm{CH} 1$ & 0.58675 & 0.58588 & 0.58674 & 0.58519 & 0.58635 \\
\hline \multirow{2}{*}{100.552} & $\mathrm{CH} 0$ & 100.555 & 100.553 & 100.555 & 100.552 & 100.551 \\
\hline & $\mathrm{CH1}$ & 100.555 & 100.552 & 100.554 & 100.555 & 100.556 \\
\hline
\end{tabular}

(b)

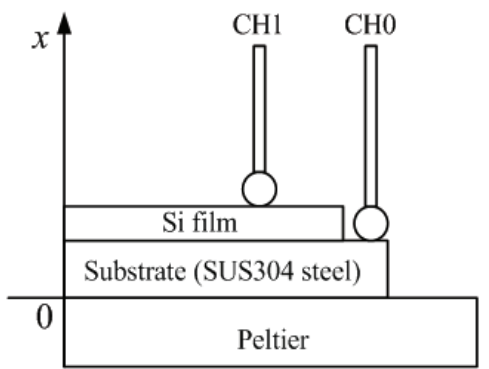

\begin{tabular}{|c|c|c|c|c|c|c|}
\hline \multicolumn{2}{|c|}{$\begin{array}{l}\text { Applied } \omega \\
\text { in peltier }(\mathrm{Hz})\end{array}$} & 1 & 2 & 3 & 4 & 5 \\
\hline 0.586 & $\mathrm{CH} 0$ & 0.58625 & 0.58528 & 0.58712 & 0.58621 & 0.58642 \\
\hline & $\mathrm{CH1}$ & 0.58668 & 0.5871 & 0.58523 & 0.58647 & 0.58549 \\
\hline 100.552 & $\mathrm{CH} 0$ & 100.552 & 100.554 & 100.555 & 100.552 & 100.556 \\
\hline & $\mathrm{CH} 1$ & 100.553 & 100.554 & 100.552 & 100.552 & 100.554 \\
\hline $\begin{array}{l}\text { Applied } \omega \\
\text { in peltier }(H\end{array}$ & & 6 & 7 & 8 & 9 & 10 \\
\hline 0.586 & $\mathrm{CH} 0$ & 0.58568 & 0.58688 & 0.58703 & 0.58635 & 0.58612 \\
\hline & CH1 & 0.58675 & 0.58588 & 0.58674 & 0.58519 & 0.58635 \\
\hline 100.552 & $\mathrm{CH} 0$ & 100.555 & 100.553 & 100.555 & 100.552 & 100.551 \\
\hline & CH1 & 100.555 & 100.552 & 100.554 & 100.555 & 100.556 \\
\hline
\end{tabular}

Fig. 6. (a) Schematic diagram of the $\mathrm{CH} 1$ and $\mathrm{CH} 0$ thermocouples arranged to measure the temperatures of the SUS 304 steel substrate at arbitrary contact positions and the $a_{3}$ values evaluated for these positions; (b) Schematic diagram of the $\mathrm{CH} 1$ and $\mathrm{CH} 0$ thermocouples arranged to measure the $\mathrm{Si}$ film and the steel substrate respectively at various contact positions and the $\mathrm{a}_{3}$ values corresponding these positions.

Instead of contact position, $\mathrm{a}_{3}$ is also obtained from the change in the electrical voltage applied to the peltier. Fig. 7(a) shows the $a_{3}$ values varying with electrical 
voltage (unit: V) for both the $\mathrm{CH} 0$ and $\mathrm{CH} 1$ in contact with the SUS304 steel substrate. The oscillating amplitude of temperature is varied with electrical voltage but frequency is kept as same as those shown in Fig. 6 . The $a_{3}$ values due to the change in electrical voltage have their magnitude almost as same as the peltier frequency, and they are extremely close to those $(0.586 \mathrm{~Hz}$ and $100.522 \mathrm{~Hz}$ ) shown in Fig. 6(a) respectively. This implies that the $a_{3}$ value remained to be invariant with the electrical voltage so long as the peltier oscillating temperature frequency is unchanged. Figure 7(b) shows the $a_{3}$ values varying with electrical voltage for the contact positions of $\mathrm{CH} 0$ and $\mathrm{CH} 1$ are as same as those shown in Fig. 6(b). These data can be averaged with $\mathrm{a}_{3}=$ $0.586 \mathrm{~Hz}$ and $100.552 \mathrm{~Hz}$ respectively too. The provisions of Fig. 6 and Fig. 7 make the conclusion that the oscillating temperature frequency $a_{3}$ created in the specimen, so long as the frequency of oscillating temperature applied to the peltier, remains to be the same

(a)

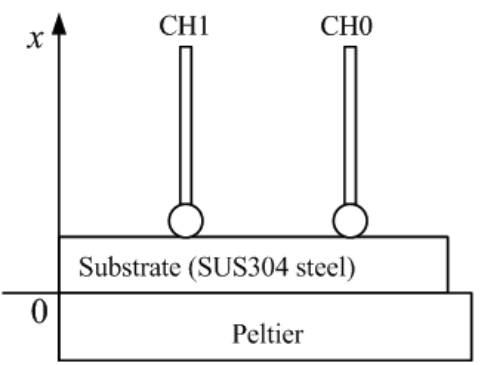

\begin{tabular}{|c|c|c|c|c|c|c|}
\hline \multicolumn{2}{|c|}{$\begin{array}{c}\text { Applied } \omega \\
\text { in peltier (Hz) }\end{array}$} & & & & \\
\hline \multirow{2}{*}{0.586} & $\mathrm{CH} 0$ & 0.58625 & 0.58528 & 0.58675 & 0.58588 & 0.58674 \\
\cline { 2 - 7 } & $\mathrm{CH} 1$ & 0.58703 & 0.58635 & 0.58612 & 0.58647 & 0.58549 \\
\hline \multirow{2}{*}{100.552} & $\mathrm{CH} 0$ & 100.552 & 100.552 & 100.554 & 100.555 & 100.556 \\
\cline { 2 - 7 } & $\mathrm{CH} 1$ & 100.553 & 100.555 & 100.553 & 100.555 & 100.554 \\
\hline
\end{tabular}

(b)

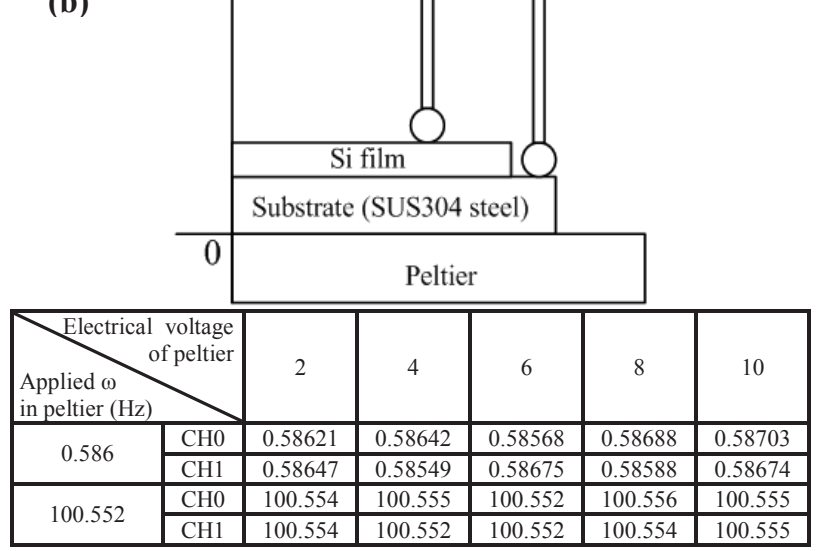

Fig. 7. (a) Schematic diagram of the $\mathrm{CH} 1$ and $\mathrm{CH} 0$ thermocouples arranged to measure the temperatures of the SUS 304 steel substrate at various electrical voltages and the $a_{3}$ values evaluated for these voltages; (b) Schematic diagram of the $\mathrm{CH} 1$ and $\mathrm{CH} 0$ thermocouples arranged to measure the $\mathrm{Si}$ film and the steel substrate respectively at various electrical voltage and the $\mathrm{a}_{3}$ values corresponding these voltages.

constant, irrespective of the applied electrical voltage and the contact positions of thermocouples. This characteristic is certainly valid for the temperature measurements using the lock-in amplifier.

The wave pattern of the reference signal can be set by the thermal-electrical data processing system with a lock-in amplifier in a flexible way; and the reference frequency in the present study is taken as a variable in order to achieve phase differences varying with the frequency. After processing by the lock-in amplifier, an oscillating temperature profile with its frequency $(\omega)$ as same as that of the reference signal can be achieved. The amplitudes of these two temperature curves in Fig. 3 may be different; however, the phase change due to the temperature waves passing through the film with a thickness of $d$ is determined equal to the difference in the time of any two adjacent peaks on these two curves. With the change in frequency at various values, the experimental data for the phase difference between the two thermocouples can be expressed as a function of $\sqrt{\omega}$. A regression of these data have been made to achieve the slope value (b). This $b$ value will be employed to determine the thermal diffusivity $(\alpha)$ of this film. In the present study, temperature profile was processed by the lock-in amplifier with a reference signal in a rectangular pattern. This temperature profile is presented to have the same frequency as that of the reference signal. It should be stressed that the noises in the original temperature signals partly attributable to the unavoidable thermal drift in the measurements can be removed from the use of the lock-in amplifier. Before the measurements, specimen's temperature in the equilibrium state (steady state), as Fig. 8 shows, was demanded.

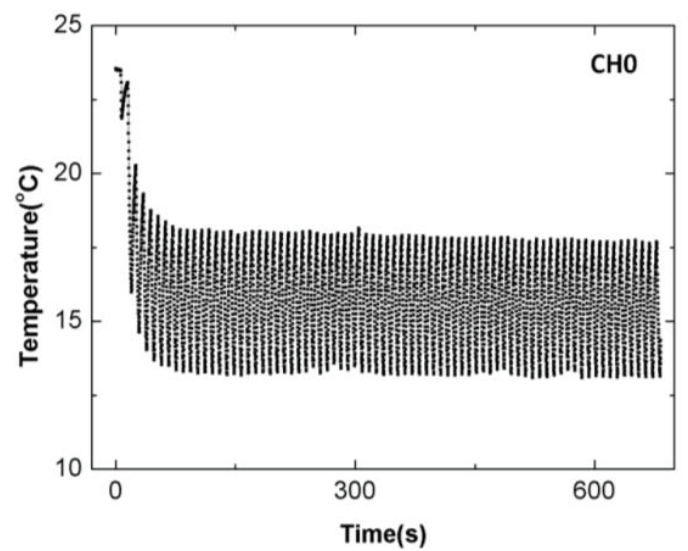

Fig. 8. Oscillating temperature profile obtained at thermally equilibrium state.

In the present study, three kinds of specimen with different coating film(s) were prepared. The SS304 stainless steel with a thickness of $5 \mathrm{~mm}$ was commonly used as the substrate of these three specimens. Al and $\mathrm{Si}$ films with a thickness of $200 \mathrm{~nm}$ were deposited on the steel substrate respectively by the technique of electron beam evaporation (EBE, VT1-10CE, Japan) with a deposition rate of $0.2 \mathrm{~nm} / \mathrm{s}$. In order to remain a small blank area in the steel substrate without the film coating, this area was stuck by an adhesive tape before conducting the thin film coating. The third type of 
specimen was provided by a research institution. The Sifilm with a thickness of $200 \mathrm{~nm}$ was deposited as the NI-P buffer layer by the technique of magnetron sputtering deposition first, then an indium tin oxide (ITO) film with a thickness of $200 \mathrm{~nm}$ was deposited successively as the top layer of the multi-layered specimen. This layer is deposited intermittently such that a periodic blank area of the buffer layer (Si(N-I-P) layer) was formed between any two ITO circular plateaus. The thermal diffusivity $(\alpha)$ of the top layer was able to determine if two thermocouples were placed to contact with the ITO film and one thermocouple was placed in the blank area of the buffer layer.

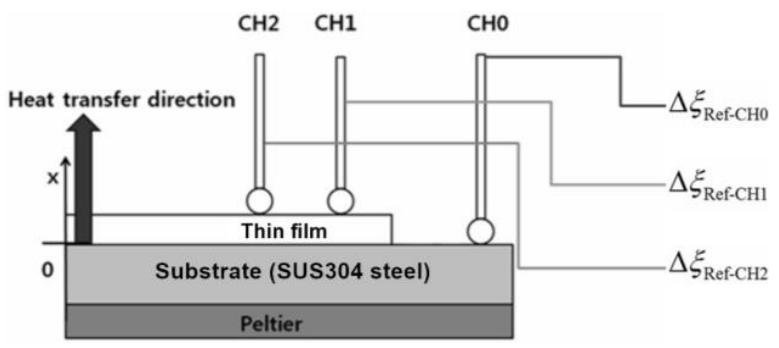

Fig. 9. Schematic diagram presented to show the placements of the three thermocouples adopted in the present study.

In the present study, the $\mathrm{CHO}$ thermocouple was arranged to contact with the steel substrate or the $\mathrm{Si}$ buffer layer (200 $\mathrm{nm}$ in thickness), and the other two thermocouples, $\mathrm{CH} 1$ and $\mathrm{CH} 2$, were arbitrary positioned to contact with the top surface of the measured film. As Fig. 9 is shown as an example, the temperature signals of $\mathrm{CH} 0$ were processed by the lock-in amplifier to obtain the phase difference $\Delta \xi_{\text {Ref-Ch0 }}$ from the reference signal. By the similar way, $\Delta \xi_{\mathrm{Ref}-\mathrm{Ch} 1}$ and $\Delta \xi_{\mathrm{Ref}-\mathrm{Ch} 2}$ are able to achieve via the channels of $\mathrm{CH} 1$ and $\mathrm{CH} 2$ respectively. Define $\phi_{\mathrm{CH} 0-\mathrm{CH} 1}$ as

$$
\phi_{\mathrm{CH} 0-\mathrm{CH} 1} \equiv \phi_{\text {Ref }-\mathrm{CH} 0}-\phi_{\text {Ref }-\mathrm{CH} 1}
$$

then, $\phi_{\mathrm{CH} 0-\mathrm{CH} 1}$ represents the phase change (lag) of temperature waves due to the thin film with a thickness of $\mathrm{d}$, it is created between the $\mathrm{CH} 0$ and $\mathrm{CH} 1$ channels. Similarly, $\phi_{\mathrm{CH} 0-\mathrm{CH} 2}$ can be achieved following the same method.

By changing the frequency $(\omega)$ of the reference signal, the phase differences were measured as a function of $\sqrt{\omega}$. Figure 10(a) and Fig. 10(b) show the experimental data of $\phi_{\mathrm{CH}-\mathrm{CH} 1}$ and $\phi_{\mathrm{CH} 0-\mathrm{CH} 2}$ varying with $\sqrt{\omega}$ respectively for the specimen with an $\mathrm{Al}$ film as the top layer. The $\omega$ is varying in a range of $0.586 \mathrm{~Hz}$ to $100.522 \mathrm{~Hz}$. These data can be regressed well by a straight line with an adj. $\mathrm{R}^{2}$ value higher than 0.96 . These two straight lines are not passing through the origin point, but rather intercepting the ordinate with a value of $a$. The slope of the straight line is denoted as $b$. Define $y$ as the temperature phase change $\left(\phi_{\mathrm{CH} 0-\mathrm{CH} 1}\right.$ or $\left.\phi_{\mathrm{CH} 0-\mathrm{CH} 2}\right)$ and $\mathrm{x}$ as the $\sqrt{\omega}$ parameter. Then, the data of phase difference can be expressed well in a form of $y=a$ $+b x=a+b \sqrt{\omega}$. Taking the differentiations of $\phi=d \sqrt{\omega / 2 \alpha}$ and $\phi=a+b \sqrt{\omega}$ with respect to $\sqrt{\omega}$ obtains

$$
\frac{\partial \phi}{\partial \omega}=\frac{\mathrm{d}}{\sqrt{2 \alpha}}=\frac{\partial(\mathrm{a}+\mathrm{b} \sqrt{\omega})}{\partial \sqrt{\omega}}=\mathrm{b}
$$
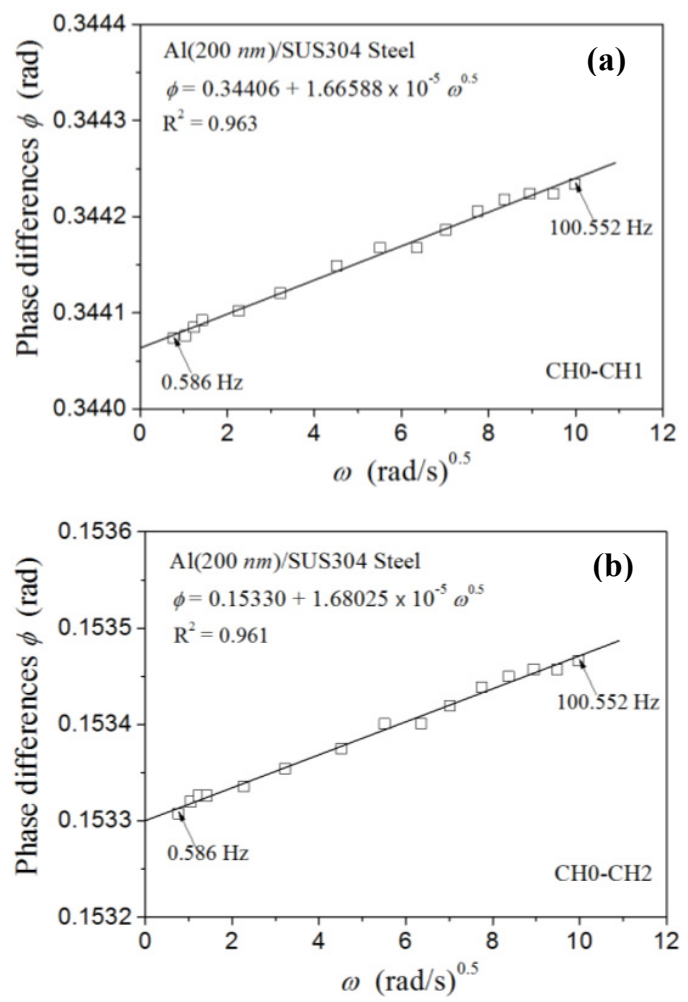

Fig. 10. Variations of (a) $\phi_{\mathrm{CH}-\mathrm{CH} 1}$; (b) $\phi_{\mathrm{CHO}-\mathrm{CH} 2}$ with $\sqrt{\omega}$ for the $\mathrm{Al} /$ steel specimen.

Eq. (7) can be rewritten to obtain the thin-film thermal diffusivity $(\alpha)$ as

$$
\alpha=\frac{d^{2}}{2 b^{2}}
$$

if the slope (b) of the straight line is available. By the method mentioned above, $\alpha_{\mathrm{CH}-\mathrm{CH} 1}$ and $\alpha_{\mathrm{CHO}-\mathrm{CH} 2}$ values are obtained as $7.21 \times 10^{-5} \mathrm{~m}^{2} / \mathrm{s}$ and $7.08 \times 10^{-5} \mathrm{~m}^{2} / \mathrm{s}$, respectively. These two $\alpha$ values are seen within the reported range, $(6.8 \sim 9) \times 10^{-5} \mathrm{~m}^{2} / \mathrm{s}$ [8]. 


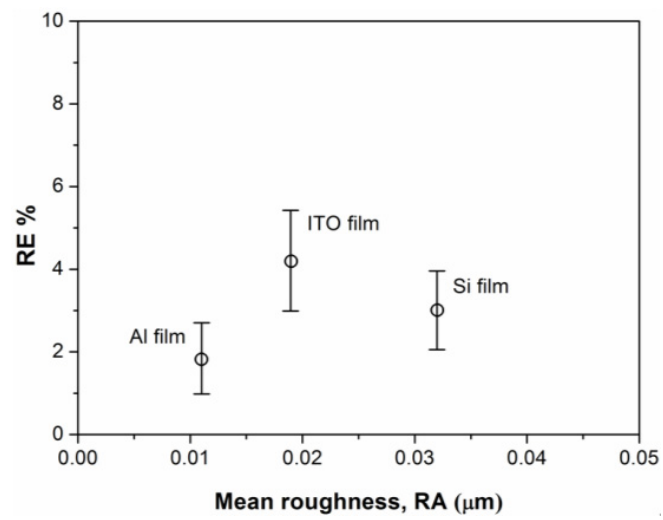

Fig. 11. The relative errors of the thermal diffusivity varying with the surface roughness of the three films.

The $\phi_{\mathrm{CH} 0-\mathrm{CH} 1}$ and $\phi_{\mathrm{CH} 0-\mathrm{CH} 2}$ v.s. $\sqrt{\omega}$ regressions for the Si/SUS304 Steel specimen have been obtained following the same way. Two slopes, $\mathrm{b}=1.67 \times 10^{-5}(\mathrm{~s} \cdot \mathrm{rad})^{0.5}$ and $\mathrm{b}=1.68 \times 10^{-5}(\mathrm{~s} \cdot \mathrm{rad})^{0.5}$, are obtained respectively. The thermal diffusivities corresponding to these two $\mathrm{b}$ values are $6.07 \times 10^{-5} \mathrm{~m}^{2} / \mathrm{s}$ and $5.89 \times 10^{-5} \mathrm{~m}^{2} / \mathrm{s}$, respectively. Quite small difference between the two $\alpha$ values is believed in relation to the surface roughness difference of the Si film, and it will be discussed in later section.

Table 1. The thermal diffusivities of the three films including the predictions by the current method and the reported data.

\begin{tabular}{|c|c|c|}
\hline Specimen & $\begin{array}{c}\text { Current } \alpha \text { results } \\
\left(\mathrm{m}^{2} / \mathrm{s}\right)\end{array}$ & $\begin{array}{l}\text { Reported data } \\
\left(\mathrm{m}^{2} / \mathrm{s}\right)\end{array}$ \\
\hline $\begin{array}{c}\text { ITO (200 } \\
\mathrm{nm}) / \text { Si/Steel } \\
(1)\end{array}$ & $3.41 \times 10^{-6}$ & \multirow{2}{*}{$\begin{array}{c}(1.5 \sim 2.3) \times 10^{-6} \\
{[17]}\end{array}$} \\
\hline $\begin{array}{c}\text { ITO (200 } \\
\mathrm{nm}) / \mathrm{Si} / \text { Steel } \\
(2)\end{array}$ & $3.27 \times 10^{-6}$ & \\
\hline $\begin{array}{c}\text { Si }(200 \mathrm{~nm}) / \text { Steel } \\
(1)\end{array}$ & $6.07 \times 10^{-5}$ & \multirow{2}{*}{$\begin{array}{c}(0.95 \sim 8.2) \times 10^{-5} \\
{[8]}\end{array}$} \\
\hline $\begin{array}{c}\mathrm{Si}(200 \mathrm{~nm}) / \text { Steel } \\
\text { (2) }\end{array}$ & $5.89 \times 10^{-5}$ & \\
\hline $\begin{array}{c}\mathrm{Al}(200 \mathrm{~nm}) / \text { Steel } \\
\text { (1) }\end{array}$ & $7.21 \times 10^{-5}$ & \multirow{2}{*}{$\begin{array}{c}(6.8 \sim 9) \times 10^{-5} \\
{[8]}\end{array}$} \\
\hline $\begin{array}{c}\mathrm{Al}(200 \mathrm{~nm}) / \text { Steel } \\
\text { (2) }\end{array}$ & $7.08 \times 10^{-5}$ & \\
\hline
\end{tabular}

The $\phi_{\mathrm{CHO}-\mathrm{CH} 1}$ and $\phi_{\mathrm{CH} 0-\mathrm{CH} 2}$ values of the ITO film as a function of $\sqrt{\omega}$ for the ITO/Si/Steel specimen have been obtained respectively too. The $b$ values for these two sets of $\phi$ values are evaluated to be $7.65 \times 10^{-5}(\mathrm{~s} \cdot \mathrm{rad})^{0.5}$ and $7.81 \times 10^{-5}(\mathrm{~s} \cdot \mathrm{rad})^{0.5}$. The thermal diffusivities, $3.41 \times 10^{-6} \mathrm{~m}^{2} / \mathrm{s}$ and $3.27 \times 10^{-6}$ $\mathrm{m}^{2} / \mathrm{s}$, are thus determined respectively. These two $\alpha$ values are quite close each other. The reported data of $\alpha$ are varying in a range of $(1.5 \sim 2.3) \times 10^{-6} \mathrm{~m}^{2} / \mathrm{s}$ [21]. Therefore, the two $\alpha$ values are somewhat outside of the reported range. This difference can be attributable to several factors including the different chemical compositions and microstructures of the ITO film prepared by different deposition techniques. The $\alpha$ values are achieved from the measures at two different positions of the top surface in the same film. Small difference in these two $\alpha$ values are thus due to different surface roughness at different position of the same film. Table 2 shows the mean surface roughness (Ra) of the three kinds of thin film and the SUS304 steel substrate. Difference in the thermal diffusivity between $\alpha_{\text {Сно-СН1 }}$ and $\alpha_{\mathrm{CH} 0-\mathrm{CH} 2}$ is found to be related to the surface roughness of the thin film as the top layer. However, it is the relative error (RE) of thermal diffusivity defined as $\mathrm{RE} \equiv\left|\alpha_{\mathrm{CH}-\mathrm{CH} 1}-\alpha_{\mathrm{CH}-\mathrm{CH} 2}\right| / \frac{1}{2}\left(\alpha_{\mathrm{CH}-\mathrm{CH} 1}+\alpha_{\mathrm{CHO}-\mathrm{CH} 2}\right)$, rather than the value of $\left|\alpha_{\mathrm{CH}-\mathrm{CH} 1}-\alpha_{\text {СHO-CH2 }}\right|$, becomes more meaningful to establish its relationship with the thin film roughness $(\mathrm{Ra})$. Figure 11 shows the RE values as a function of $\mathrm{Ra}$ for these three thin films. The three $\mathrm{RE}$ values show the sequence that $(\mathrm{RE}=1.8 \pm 0.86 \%)_{\mathrm{Al}}$ film $<(\mathrm{RE}=2.97 \pm 0.91 \%)_{\mathrm{Si} \text { film }}<(\mathrm{RE}=4.25 \pm 1.10 \%)_{\mathrm{ITO}}$ film. It can be noticed that small RE occurred in these three film materials with their value lower than $6 \%$.

Table 2. Surface roughnesses of the three films and the SUS304 steel substrate.

\begin{tabular}{|c|c|}
\hline Thin film/substrate material & Roughness, Ra $(\mu \mathrm{m})$ \\
\hline ITO & 0.019 \\
\hline $\mathrm{Si}$ & 0.032 \\
\hline $\mathrm{Al}$ & 0.011 \\
\hline SUS304 steel & 0.073 \\
\hline
\end{tabular}

\section{Conclusions}

The thermal-electrical data acquisition and processing system without lock-in amplifier is efficiently employed to examine the effects of the electrical voltage applied to the peltier and the contact position of thermocouples on the frequency of the oscillating temperature solution created at thin coating films. As long as a constant frequency is fixed in the oscillating temperature of the peltier, the oscillating frequency of oscillating temperature at various layers remains to be the same constant, irrespective of the changes in the electrical voltage of the peltier and the contact position of thermocouples. The above characteristic is also valid for the thermal-electrical system including the lock-in amplifier, which is applied to evaluate the thermal diffusivity of coating films. The present method provides an efficient way to determine the film thermal diffusivity via the measures of the phase difference of onedimensional oscillating temperature wave across the film with a thickness $d$. With the proper design of the peltier heating system, the phase difference $(\phi)$ results are proved to be linearly proportional to $\sqrt{\omega}$ ( $\omega$ : temperature wave frequency) from the regression of the experimental data of different thin film material. The 
thin-film thermal diffusivity can be determined accurately if the slope of the regressed line and the film thickness are available. The relative errors of thermal diffusivity resulting from different thermocouple contact position for these three coating films with different surface roughness are quite small $(<6 \%)$. The experimental results of thin film thermal diffusivity obtained from the current technique are consistent with those reported in the literatures.

This work has been granted by Frontier Materials and Micro/Nano Science and Technology Center, National Cheng Kung University, Taiwan, R.O.C.

\section{References}

1. M.J. Adams, C.F. Kirkbright, Analyst, 102, 281 (1977)

2. M.J. Adams, C.F. Kirkbright, Analyst, 102, 678 (1977)

3. A.J. Ångström, Phil. Mag., 26, 161 (1863)

4. R.W. King, Phys. Rev., 6, 437 (1915)

5. B. Abeles, G.D. Cody, D.S. Beers, J. Appl. Phys., 31, 1585 (1960)

6. Y.S. Touloukian, R.W. Powell, C.Y. Ho, M.C. Nicolasu, Thermal Diffusivity (IFI/Plenum, New York, 1973)

7. M.B. Salamon, P.R. Garnier, B. Golding, E. Buehler, J. Phys. Chem. Solids, 35, 851 (1974)

8. I. Hatta, Y. Sasuga, R. Kato, A. Maesono, Rev. Sci Instrum., 56, 1643 (1985)

9. D.G. Cahill, M. Katiyar, J.R. Abelson, Phys. Rev. B 50, 6077 (1994)

10. W. Czarnetzki, W. Roetzel, Internat. J. of Thermophys., 16, 413 (1995)

11. X. Zhang, C.P. Grigoropoulos, Rev. Sci. Instrum., 66, 1115 (1995)

12. X.Y. Yu, L. Zhang, G. Chen, Rev. Sci. Instrum., 67, 2312 (1996)

13. E. Jansen, E. Obermeier, J. Micromech. Microeng., 6, 118 (1996)

14. D.G. Cahill, Review Article, Microscale Thermophys. Egin., 1, 85 (1997)

15. D.M. Bhusari, C.W. Teng, K.H. Chen, S.L. Wei, L.C. Chen, Rev. Sci. Instrum., 68, 4180 (1997)

16. T. Yamane, Y. Mori, S.I. Katayama, M. Todoki, J. Appl. Phys., 82, 1153 (1997)

17. Y.D. Chung, A.P. Chojnacka, C.T. Avedisian, R. Raj, Acta Mater., 45, 2983 (1997)

18. N. Taketoshi, T. Baba, A. Ono, Measur. Sci. and Techn., 12, 2064 (2001)

19. A. Muscio, P.G. Bison, S. Marinetti, E. Grinzato, Internat. J. of Thermal Sci., 43, 453 (2004)

20. A. Mathew, J. Ravi, K.N. Madhusoodanan, K.P.R. Nair, T.M.A. Rasheed, Appl. Surf. Sci., 277, 410 (2004)
21. T. Ashida, A. Miyamura, N. Oka, Y. Sato, T. Yagi, J. Appl. Phys., 105, 73709 (2009)

22. T.R. Kakuda, A.M. Limarga, T.D. Bennett, D.R. Clarke, Acta Mater., 57, 2583 (2009)

23. G.I. Athanasopoulos, E. Svoukis, M. Pervolaraki, R. Saint-Martin, A. Revcolevschi, J. Giapintzakis, Thin Solid Films, 518, 4684 (2010)

24. J.E.D. Albuquerque, C. Giacomantonio, A.G. White, P. Meredith, Appl. Phys. Lett., 87, 061920 (2005)

25. B. Raneesh, H. Soumya, J. Philip, N. Kalarikkal, J. Alloys Comp., 579, 243 (2013)

26. P.H.M. Böttger, A.V. Gusarov, V. Shklover, J. Patscheider, M. Sobiech, Int. J. Therm. Sci., 77, 75 (2014)

27. H. Wang, Q.H. Qin, Appl. Math. Compt., 268, 311 (2015)

28. B. Weidenfeller, S. Kirchberg, Compos., Part B 92, 133 (2016)

29. R. Verma, C. Lal, I.P. Jain, J. Mater. Res. Technol., 3, 257 (2014) 